\title{
Respuesta morfológica de cuatro híbridos comerciales de maíz (Zea mays L.) por fertilización edáfica y edáfica-foliar
}

\author{
Morphologic Response of Four Commercial Hybrids of Maize (Zea mays $L$.) by Soil and Soil-leaf Fertilization. \\ Luigy Barragán Rosadoㄹ, Carmen Rosero Guillen ${ }^{1}$, David Campi Ortiz, Javier Auhing Arcos ${ }^{1}$, Hayron Canchignia Martínez². \\ ${ }^{1}$ Facultad de Ciencias Agrarias, Universidad Técnica Estatal de Quevedo, Campus Ing. Manuel Haz Álvarez, $\mathrm{km} 1.5$ vía a \\ Santo Domingo de los Tsáchilas.EC.120501.Quevedo,Ecuador.luigybarragan@gmail.com; carmen.rosero@uteq.edu.ec; \\ javier.auhing@uteq.edu.ec \\ ${ }^{2}$ Área de Microbiología Molecular del Departamento de Biotecnología, Facultad de Ciencias Agrarias, Escuela de Agronomía, \\ Universidad Técnica Estatal de Quevedo, Campus Ing. Manuel Haz Álvarez, km 1.5 vía a Santo Domingo de los Tsáchilas. \\ EC.120501. Quevedo, Ecuador. \\ Autor de correspondencia: Hayron Fabricio Canchignia Martínez; hfcanma@gmail.com; hcanchignia@uteq.edu.ec
}

Rec.: 24.07.2017. Acept.: 13.11.2017 Publicado el 2 de Julio de 2018

\section{Resumen}

$\mathrm{E}_{\mathrm{pex}}^{1}$ rendimiento del cultivo de maíz en Ecuador se encuentra por debajo del potencial genético de los híbridos cultivados. Entre los factores limitantes se encuentra la deficiencia de micronutrientes. La fertilización foliar es una práctica empleada para corregir las deficiencias nutricionales no suministradas por la fertilización edáfica. El objetivo fue evaluar la eficiencia de los fertilizantes foliares en los caracteres morfológicos de los híbridos: H.Das-3383, 2B-604, Insignia-105 y Dekalb-7500. Se empleó un diseño de bloques al azar, con ocho tratamientos. La fertilización foliar acelera el proceso de formación de órganos florales al híbrido 2B-604 a DFM y DFF con 50 y 53 días, también mejora la AP y AIM, con 244 y $146.67 \mathrm{~cm}$. El DM y LM es incrementada con 4.9 y 18,91 cm. Los híbridos 2B-604 e Insignia-105 alcanzaron los mayores rendimientos con 7,19 y $7,18 \mathrm{t} / \mathrm{ha}^{-1}$ respectivamente. El programa de fertilización edáfica+foliar, realza el potencial genético de los híbridos 2B604 e Insignia-105, por la asimilación de nutrientes a través de sus hojas

Palabras clave: potencial genético, micronutrientes, fertilización foliar.

\begin{abstract}
Corn crop yields are below the genetic potential of hybrids grown in Ecuador. Among the limiting factors is micronutrient deficiency. Foliar fertilization is a practice used to correct nutritional deficiencies not provided by edaphic fertilization. The objective was to evaluate the efficiency of foliar fertilizers in the morphological characters of the hybrids: H.Das-3383, 2B-604, Insignia-105 and Dekalb-7500. A randomized block design was used, with eight treatments. The foliar fertilization accelerates the process of formation of floral organs to the hybrid 2B-604 to DFM and DFF with 50 and 53 days, also improves the AP and AIM, with 244 and $146.67 \mathrm{~cm}$. The DM and LM is increased with 4.9 and 18.91 $\mathrm{cm}$. The hybrids 2B-604 and Insignia-105 reached the highest yields with 7.19 and $7.18 \mathrm{t} / \mathrm{ha}^{-1}$ respectively. The program of edaphic + foliar fertilization, enhances the genetic potential of the hybrids 2B-604 and Insignia-105, by the assimilation of nutrients through its leaves.
\end{abstract}

Keywords: genetic potential, micronutrients, foliar fertilization. 


\section{Introducción}

$\mathrm{L}$ a fertilización foliar es una alternativa complementaria a fertilización edáfica, para incrementar los rendimientos de cultivos por la aportación de macro y micronutrientes que son rápidamente asimilables durante el desarrollo del maíz (Bautista et al., 2002). La fertilización foliar con moléculas húmicas incrementa la retención de agua $\mathrm{y}$ el proceso fotosintético en hojas (Fu Jiu et al., 1995). Además, aumenta el sistema radicular y el indexe de área foliar (Figliolia et al., 1994). Donde los fertilizantes foliares favorece a las plantas en división y desarrollo celular de tejido meristematicos y la aceleración en madurez (Zeidan et al., 2010), por la presencia de ácido indol-3-acetico (AIA) en los abonos foliares que incrementa en longitud y número de hojas y rendimiento en grano (Thavaprakaash et al., 2007).

El cultivo de Zea mays L. tiene demandas nutricionales altas, especialmente de nitrógeno $(\mathrm{N})$, potasio $(\mathrm{K})$ y fósforo $(\mathrm{P})$, nutrientes esenciales de la mayoría de fertilizantes químicos, en forma individual o combinados en fórmulas (Dunja, 2000). Además de N, P y K, las plantas necesitan de otros elementos del suelo, requeridos en menor proporción como calcio (Ca), magnesio $(\mathrm{Mg})$ y azufre $(\mathrm{S})$ y los denominados micronutrientes componentes de abonos foliares (Dunja, 2000).

Los fertilizantes foliares con contenidos de P y Zn, incrementa el desarrollo de los caracteres morfológicos en altura y diámetro del maíz (Bukvić et al., 2003), al mejorar este aspecto fisiológico de la planta beneficia con el aumento en productividad, mazorcas de mayor longitud en comparación de fertilización edáfica con N, P, K (Potarzycki y Grzebisz, 2009). Donde $P$ en fertilizantes foliares aumenta el número de semillas, biomasa y peso de semillas (Khaliq y Sanderz, 2000). En respuesta de la fertilización foliar por ácido indolbutírico (IBA) y ácido giberélico (GA) en maíz, incrementando en número de semillas por fila por mazorca (Ghodrat et al., 2012). Las auxinas juegan un importante rol en la formación de granos y el aumento de transporte de compuestos metabólicos a los granos (Prochazka, 1978).

En Ecuador existen 310787.8 ha, destinado a la producción de maíz duro con una producción promedio de $4.8 \mathrm{t} / \mathrm{ha}$ (Monteros, 2015). Los problemas al rendimiento del maíz en la provincia, se originan por fertilizaciones inadecuadas o empíricas que ocasionan un desbalance en el crecimiento vegetativo de la planta (Shahid et al., 2014). Esta investigación tiene como objetivo evaluar el comportamiento de dos programas de fertilización en la mejora de las características morfológicas en híbridos comerciales de maíz, H.Das-3383, 2B-604, Insignia-105 y Dekalb-7500.

\section{Materiales y métodos}

La investigación se realizó en la Finca "El Azúcar” del cantón Valencia, provincia de Los Ríos (Ecuador), en las coordenadas geográficas: Norte $0^{\circ} 96^{\prime} 67^{\prime \prime}$ y Oeste 79³8' 42", a $173 \mathrm{msnm}$. El clima tiene las siguientes características: temperatura promedio anual de 24,89 oC, precipitación promedio anual de $2554,94 \mathrm{~mm}$, humedad relativa promedio de $83.24 \%$ y 895.48 horas luz al año. Los estudios se efectuaron en la época seca del año 2015.

Se utilizaron cuatro híbridos comerciales de maíz: H.Das-3383, 2B-604, Insignia-105 y Dekalb-7500. Se empleó la fertilización propuesta por el Programa de maíz de la Estación Experimental Tropical Pichilingue (EETP) del Instituto Nacional de Investigaciones Agropecuarias (INIAP) (Tabla 1). La fertilización se realizó con: Mezclafix (N 16, P 10, S 2); Urea 46 \%; BIOTEK (Citoquininas 2197.95, Auxinas 34.70, Giberelinas 33,50 ppm, N 7.4, P 14.7, K 15.5, Ca 3.7, Mg 6.2, Fe 28.3, Zn 35.7, Mn 14.8, Cu 7.2, B 5.3); Raizplant (N 4.8, P 22, K 15.5, Mg 0.3, S 0.4 \%), B 150 ppm, Fitohormonas 500 ppm; ECO-HUM DX (Humatos, fulvatos y ácido himatomelánico 12, NH4+NO3 8, K 6, P2O5 6, MgO $0.5 \%$ ), B 20 ppm; EVERGREEN (Citoquininas 90, Auxinas 40, Giberelina 90, ppm), (N 7, P7, K 7, B 0.024, Fe 0.013 Mn $0.05, \mathrm{Mg} 0.018, \mathrm{Zn} 0.0009$ \%). En las parcelas control no se realizaron aplicaciones de fertilizantes, el control de plagas y/o enfermedades en caso de ser necesario para: Spodoptera frugiperda (gusano cogollero), Phyllachora maydis (mancha de asfalto), Exserohilum turcicum (tizón foliar). El manejo químico de malezas e insectos se efectuó de acuerdo al plan detallado en la Tabla 2.

Tabla 1. Programa de fertilización edáfica y edáfica+foliar.

\begin{tabular}{lcccc}
\hline \multicolumn{5}{c}{ Días a fertilizar } \\
\hline Fertilización & $\mathbf{0}$ & $\mathbf{1 2 - 1 4}$ & $\mathbf{1 8 - 2 0}$ & $\mathbf{3 5 - 4 0}$ \\
\hline Edáfica & Mezclafix $200 \mathrm{~kg} / \mathrm{ha}$ & & Urea $46 \% 150 \mathrm{~kg} / \mathrm{ha}$ & Urea $46 \% 200 \mathrm{~kg} / \mathrm{ha}$ \\
\hline Edáfica+foliar & Mezclafix $200 \mathrm{~kg} / \mathrm{ha}$ & $\begin{array}{c}\text { Biotek-200 } \mathrm{ml} / 200 \mathrm{~L}+ \\
\text { Raizplant }(500) \\
1 \mathrm{~L} / 200 \mathrm{~L} \mathrm{x} \mathrm{ha}\end{array}$ & $\begin{array}{c}\text { Urea } 46 \% 150 \mathrm{~kg} / \mathrm{Ha} \\
+ \text { ECO-HUM DX } \\
+ \text { EVERGREEN } 1 \mathrm{~L} / \mathrm{ha}\end{array}$ & $\begin{array}{c}\text { Urea } 46 \% 200 \mathrm{~kg} / \mathrm{ha} \\
+ \text { ECO-HUM DX } \\
+ \text { EVERREEN } 1 \mathrm{~L} / \mathrm{ha}\end{array}$ \\
\hline
\end{tabular}


Tabla 2. Plan de manejo al control de insectos y malezas realizado en los híbridos de maíz

\begin{tabular}{|c|c|c|}
\hline$\left(\right.$ dde $\left.^{*}\right)$ & Producto & Plagas \\
\hline Protección de Semilla & Semevin10 cc/kg de semilla (Thiodicarb) & Insectos del suelo \\
\hline Siembra & $\begin{array}{l}\text { Glifosato } 2 \mathrm{~L} / \mathrm{ha} \\
\text { + Atrazina }(0,9 \mathrm{~kg} / \mathrm{mz}) \\
\text { + 2,4-D Dimethylamine } 0.5 \mathrm{~L} / \mathrm{ha}\end{array}$ & Malezas \\
\hline A los 5 dde. & Lorsban 4E (Chlorphyrifos) $(1.5 \mathrm{~L} / \mathrm{ha})$ & Spodoptera, Diatraea. Spp. \\
\hline A los 15 dde. & Lorsban 4E $®(1.5 \mathrm{~L} / \mathrm{ha})$ & Spodoptera, Diatraaea. Spp. \\
\hline Después de 30 dde. & Deshierba manual & Malezas \\
\hline Después de 30 dde. & $\begin{array}{l}\text { Kuik Metomil (S-methyl } \\
\text { N-methylcarbamoyloxy thioacetimidate) } 1 \mathrm{~L} / \text { ha }\end{array}$ & Spodoptera \\
\hline
\end{tabular}

* dde: Días después de la emergencia

\section{Descripción del experimento}

La investigación se desarrolló en una superficie de 240 $\mathrm{m}^{2}$. Las parcelas experimentales fueron de $4.0 \times 2.5 \mathrm{~m}$, de $10 \mathrm{~m}^{2}$. La distancia entre hileras y plantas de maíz fue de $0.8 \times 0.2 \mathrm{~m}$, una densidad por hectárea de 62500 plantas. Fueron registradas las variables del desarrollo vegetativo y producción como: días de floración masculina (DFM) y floración femenina (DFF) y; altura de planta (AP), diámetro del tallo (DT) y altura de inserción de mazorca (AIM); diámetro de mazorca (DM), longitud de mazorca (LM), peso de mil semillas (PMS) y rendimiento (R). La variable días floración resulta al cuantificar el periodo transcurrido desde la siembra hasta cuando el $50 \%$ de las plantas que conforman la parcela útil hayan florecido. Las variables, longitud de mazorca $(\mathrm{cm})$, diámetro de mazorca $(\mathrm{cm})$, hileras por mazorca y peso de mil semillas (g), se evaluó por diez mazorcas seleccionadas al azar en la parcela útil de cada unidad experimental.

Para determinar el diámetro de cada mazorca se consideró su tercio medio utilizando un calibrador graduado en $\mathrm{cm}$, el peso de sus semillas y el peso de mil semillas, empleándose para el efecto una balanza Scout Pro de $2000 \mathrm{~g}$ con una precisión de \pm 0.1 . Para cuantificar estas dos últimas variables se ajustó la humedad de la semilla al $13 \%$.

El rendimiento se determinó en función de cosecha del área útil de cada parcela experimental, empleando la fórmula: RU=RA (100-HA)/ (100-HD) x (10 000/AU). Dónde: $\mathrm{RU}=$ Rendimiento por hectárea, $\mathrm{kg} ; \mathrm{RA}=$ Rendimiento actual, $\mathrm{kg} ; \mathrm{HA}=$ Humedad actual, \%; HD=Humedad deseada, \%, y $\mathrm{AU}=$ Área útil $\mathrm{m}^{2}$. Rendimiento actual: Se cosechó la totalidad de la parcela útil de maíz $8,4 \mathrm{~m}^{2}$, se desgranó y peso en kg; Humedad actual: Se tomaron 100 semillas, se las peso para luego secarlas al horno a $130^{\circ}$ C. durante dos horas y se realizó un segundo pesado La HA fue determinada haciendo uso de la siguiente formula: $\mathrm{HA}=(\mathrm{PMS} / \mathrm{PMH}) \times 100$. Dónde: HA = Humedad actual, \%; PMS= Peso de la muestra seca; $\mathrm{PMH}=$ Peso de la muestra húmeda. Humedad deseada: Se ajustó la humedad de semilla a la deseada en el mercado (13\%).

\section{Diseño experimental}

Se empleó un diseño de Bloques al Azar, con ocho tratamientos (híbridos + fertilizaciones) y cuatro controles con tres repeticiones. Los datos generados a los caracteres morfológicos, fueron sujetos a la ANOVA, los promedios fueron separados por procedimiento de comparación múltiple de Tukey SD, con un nivel de significancia de $(\mathrm{P} \leq 0.05)$, empleando InfoStat (Di Rienso et al., 2008).

\section{Resultados y Discución}

\section{Caracteres morfológicos al desarrollo vegetativo en híbridos de maíz}

Las aplicaciones de fertilizantes foliares empleados en la investigación como: BIOTEK, RAIZPLANT, EVERGREEN, contienen reguladores de crecimiento de tipo auxinico que aceleran el proceso de floración en maíz. La fertilización edáfica+foliar en el híbrido 2B-604 obtuvo el promedio más precoz de DFM y DFF con (50 y 53 días) respectivamente (Tabla 3). La aplicación del fertilizante foliar acelera el proceso de formación de órganos florales, estimulando la precocidad del maíz entre híbridos, acelerando sus días a floración femenino (DFF) y días a floración masculino (DFM), por efecto de la composición de macronutrientes, micronutrientes y reguladores de crecimiento que contienen los abonos foliares empleados. Kefyalew et al., (2007) manifiestan que las aplicaciones de abonos foliares con presencia de $\mathrm{P}$, estimulan las etapas de crecimiento del maíz.

Es evidente que la fertilización edáfica + foliar contribuye de manera significativa al incremento en el desarrollo vegetativo de los híbridos de maíz, la aplicación de fertilizantes foliares como EVERGREEN y BIOTECK, en su formulación contiene macro y micronutrientes que favorecieron en los caracteres morfológicos de la planta en AP y AIM. La presencia del Zn participa en los cambios morfológicos del maíz, las dosis suministradas por los fertilizantes en este trabajo mantuvieron un efecto positivo para la planta, en sus caracteres morfológicos; de los híbridos de maíz Insignia-105, Dekalb-7500 y 2B-604. Se observa un incremento para los caracteres AP, AIM, DT 
en respuesta de la aplicación del fertilizante foliar registrando diferencias significativas entre los tratamientos. Al T7 con el híbrido Insignia-105 se verifica la mayor AP con $244 \mathrm{~cm}$, al
T6 con el híbrido 2B-604 reporta la mayor AIM con 146.67 cm y el T8 con el hibrido Dekalb-7500 se observa el mayor DT con 2,55 cm (Tabla 3).

Tabla 3. Días a floración femenina y masculina, altura de planta, altura de inserción de mazorca y diámetro del tallo. En relación a fertilización edáfica y edáfica+foliar, en los híbridos: H.Das-3383; 2B-604; Insignia-105; Dekalb-7500.

\begin{tabular}{|c|c|c|c|c|c|c|}
\hline \multicolumn{7}{|c|}{ DESARROLLO VEGETATIVO } \\
\hline FERTILIZACIÓN & $\operatorname{DFM}(d)$ & DFF (d) & $\mathbf{A P}(\mathbf{c m})$ & AIM & (cm) & DT (cm) \\
\hline T1) E+H.Das-3383 & $52,67 \mathrm{ab}$ & $55,33 \mathrm{ab}$ & $200 \mathrm{abc}$ & 80.00 & $\mathrm{f}$ & $2.21 \mathrm{bc}$ \\
\hline T2) $\mathrm{E}+2 \mathrm{~B}-604$ & $53,67 \mathrm{ab}$ & $56,00 \mathrm{ab}$ & $241 \mathrm{ab}$ & 115.33 & $\mathrm{bc}$ & $1.94 \mathrm{cdf}$ \\
\hline T3) E+Insignia-105 & $56,33 \mathrm{bcd}$ & 59,00 bc & $225 \mathrm{abc}$ & 95.67 & bcdf & $2.15 \mathrm{bcd}$ \\
\hline T4) E+Dekalb-7500 & $54,67 \mathrm{abc}$ & $54,67 \mathrm{ab}$ & $198 \mathrm{bc}$ & 111.67 & bcd & $2.32 \mathrm{ab}$ \\
\hline T5) $\mathrm{E}+\mathrm{F}+$ H.Das-3383 & $52,33 \mathrm{ab}$ & $55,00 \mathrm{ab}$ & 209 abc & 83.33 & $\mathrm{df}$ & $2.07 \mathrm{bcd}$ \\
\hline T6) $\mathrm{E}+\mathrm{F}+2 \mathrm{~B}-604$ & $50,00 \mathrm{a}$ & 53,00 a & $240 \mathrm{ab}$ & 146.67 & $\mathrm{a}$ & $1.85 \mathrm{df}$ \\
\hline T7) $\mathrm{E}+\mathrm{F}+$ Insignia-105 & $54,00 \mathrm{ab}$ & $55,67 \mathrm{ab}$ & 244 a & 125.67 & $\mathrm{ab}$ & $2.28 \mathrm{ab}$ \\
\hline T8) E+F+ Dekalb-7500 & $52,33 \mathrm{ab}$ & $54,67 \mathrm{ab}$ & $240 \mathrm{ab}$ & 85.67 & cdf & $2.55 \mathrm{a}$ \\
\hline C+ H.Das-3383 & $61,33 \mathrm{de}$ & $62,33 \mathrm{~cd}$ & $190 \mathrm{c}$ & 95.33 & bcdf & $1.93 \mathrm{cdf}$ \\
\hline $\mathrm{C}+2 \mathrm{~B}-604$ & $61,67 \mathrm{de}$ & $63,00 \mathrm{~cd}$ & $189 \mathrm{c}$ & 86.67 & bcdf & $1.66 \mathrm{f}$ \\
\hline $\mathrm{C}+$ Insignia-105 & $65,00 \mathrm{e}$ & $66,33 \mathrm{~d}$ & $194 \mathrm{bc}$ & 98.00 & bcdf & $1.93 \mathrm{cdf}$ \\
\hline $\mathrm{C}+$ Dekalb-7500 & 60,33 cde & $62,67 \mathrm{~cd}$ & $189 \mathrm{c}$ & 104.67 & bcdf & $2.23 \mathrm{abc}$ \\
\hline CV(\%) & 4.09 & 4.69 & 6.87 & 10.17 & & 5.19 \\
\hline
\end{tabular}

$(\mathbf{P}<\mathbf{0 . 0 5})$ Los valores con letras similares no representan diferencias estadísticas significativas al nivel de confianza del 95 \%, por el procedimiento de comparación múltiple de Tukey. Edáfica (E); Foliar (F); Control (C); Días (d)

Según (Bukvić et al., 2003), establece que la aplicación de concentraciones altas de $\mathrm{Zn}$ a $(5 \mathrm{~kg} / \mathrm{ha})$ como abono foliar, incide en la reducción en altura del maíz. Las aplicaciones más altas de $\mathrm{Zn}$ de $(6 \mathrm{~kg} / \mathrm{ha})$ resulta en la perdida el rendimiento del maíz, cabe destacar que (Fecenko y Ložek, 1998), demuestran que en dosis entre 1.5 a $3 \mathrm{~kg}$ / ha son las óptimas para la formación de grano de maíz. Además, las aplicaciones de bio-fertilizantes a base de $\mathrm{N}$ y P (Nitrokara y Biozar) en maíz, incrementa el desarrollo en altura de 194 y $193 \mathrm{~cm}$, respectivamente (Beyranvand et al., 2013). Los abonos foliares como: BIOTEK, RAIZPLANT, ECO-HUM DX y EVERGREEN contienen micronutrientes como $\mathrm{P}$ y $\mathrm{Zn}$, que están favoreciendo al DT en maíz. Esto concuerda con Bukvić et al., (2003), observando un incremento al desarrollo del maíz en altura y diámetro con 124 y $2.03 \mathrm{~cm}$ bajo la fertilización foliar a base de P y Zn. Donde los programas de fertilización edáfica+foliar, influyerón sobre caracteres morfológicos de los tres híbridos.

La aplicación de abonos foliares en etapa de desarrollo mejoró ciertos caracteres morfológicos en los híbridos de maíz: H.Das-3383; 2B-604; Insignia-105 y Dekalb-7500. Observando respuestas distintas para cada híbrido, en Insignia-105 y Dekalb-7500 se incrementa en la variable AP por efecto de la fertilización edáfica+foliar, pero indistintamente no se observa cambio alguno para la AIM en Dekalb-7500 por fertilización edáfica (Tabla 3), esto confirma que los caracteres morfológicos responden al potencial genético de cada híbrido señalando que la fertilización foliar no puede ejercer mayores cambios en los caracteres morfológicos en los híbridos debido a la genética del mismo, pero observando abundante follaje por efecto de fertilización edáfica+foliar.

Caracteres morfológicos a productividad en híbridos de maíz Los resultados en diámetro de mazorca (DM) y longitud de mazorca (LM) se ven mejorados por la combinación del fertilizante edáfica+foliar (BIOTEK y EVERGREEN) aplicados al maíz, dentro de los micronutrientes que aportan los fertilizantes tenemos al $\mathrm{Zn}$. Observandose diferencias estadísticas entre los tratamientos. Se incrementó el DM para el T6 al híbrido 2B-604 con $4.9 \mathrm{~cm}$, en LM con los híbridos 2B-604 e Insignia-105 con 18.91 y $18.90 \mathrm{~cm}$, respectivamente. Los híbridos sin fertilización (Control), presentan los menores DM entre 3.03 y $3.76 \mathrm{~cm}$ y LM 13.02 y $16.78 \mathrm{~cm}$. Se corrobora con Potarzycki y Grzebisz (2009), las aplicaciones de abonos foliares presente el $\mathrm{Zn}$, incrementa la productividad al $18 \%$, con longitudes de mazorca de $15.72 \mathrm{~cm}$., comparados con fertilización edáfica con N-P-K. Corroborando con Suwanarit y Sestapukdee (1989), la aplicación de N como fertilizante foliar incrementa la síntesis de clorofila en hojas. Ling y Silberbus. (2002), sin fertilización foliar el maíz presenta hojas cloróticas, señalando que $\mathrm{N}$ y $\mathrm{P}$ son los principales nutrientes que suministran el principal efecto al desarrollo del maíz, a través de producción de clorofila en consecuencia sobre la fotosíntesis. Potarzycki y Grzebisz (2009), indican que al no realizar aplicaciones con abonos foliares decae la productividad con mazorcas de menor longitud hasta 13.88 centimetros.

El peso de mil semillas y rendimiento refleja un incremento para la combinación del tratamiento con fertilizante edáfica+foliar y los híbridos 2B-604 e Insignia-105, obteniendo el mayor 
peso por semillas. Los abonos foliares empleados mejoran los caracteres morfológicos del maíz, incrementando el PMS en 429 y 428.33 gramos, respectivamente, a diferencia del control sin fertilizante foliar con 368.33 y $395 \mathrm{~g}$. (Tabla 4). El incremento en semillas a PMS por aplicación de abonos foliares mejoran el potencial genético de los híbridos en estudio. Esto concuerda con Beyranvand et al., (2013), la aplicación al maíz de bio-fertilizantes (NITROKARA y BIOZARr), obtienen $40 \mathrm{~g}$. al peso de (100 semillas). El incremento en liberación de $\mathrm{P}$ aumenta la productividad semillas, biomasa y peso de semillas (Khaliq y Sanderz. 2000). Law K y Law J (2009), la fertilización edáfica con N, P y K (15: 15: 15) a $400 \mathrm{~kg} / \mathrm{ha}$, obtienen $11.62 \mathrm{~g}$. al peso (100 semillas).

Tabla 4. El comportamiento productivo a diámetro de mazorca, longitud de mazorca, peso de mil semillas, rendimiento. En relación a fertilización edáfica y edáfica+foliar, en los híbridos: H.Das-3383; 2B-604; Insignia-105; Dekalb-7500.

\section{COMPORTAMIENTO PRODUCTIVO}

\begin{tabular}{|c|c|c|c|c|}
\hline INTERACCIÓN & DM (cm) & LM (cm) & PMS (gr) & $R$ (t/ha) \\
\hline T1) E+H.Das-3383 & $4.11 \mathrm{abc}$ & $18.61 \mathrm{ab}$ & 291.67 def & 4,89 def \\
\hline T2) $\mathrm{E}+2 \mathrm{~B}-604$ & $4.45 \mathrm{ab}$ & $18.73 \mathrm{ab}$ & 368.33 bc & $6,16 \mathrm{bc}$ \\
\hline T3) E+Insignia-105 & $3.97 \mathrm{abc}$ & $18.41 \mathrm{ab}$ & $395.00 \mathrm{ab}$ & $6,61 a b$ \\
\hline T4) E+Dekalb-7500 & $4.10 \mathrm{abc}$ & $16.63 \mathrm{abc}$ & $315.00 \mathrm{de}$ & $5,29 \mathrm{de}$ \\
\hline T5) E+F+ H.Das-3383 & $4.13 \mathrm{abc}$ & $18.66 \mathrm{ab}$ & $328.33 \mathrm{~cd}$ & $5,51 \mathrm{~cd}$ \\
\hline T6) $\mathrm{E}+\mathrm{F}+2 \mathrm{~B}-604$ & $4.90 \mathrm{a}$ & $18.91 \mathrm{a}$ & $429.00 \quad \mathrm{a}$ & 7,18 a \\
\hline T7) $\mathrm{E}+\mathrm{F}+$ Insignia-105 & $4.15 \mathrm{abc}$ & $18.90 \mathrm{a}$ & 428.33 a & 7,19 a \\
\hline T8) E+F+ Dekalb-7500 & $4.17 \mathrm{abc}$ & $17.84 \mathrm{ab}$ & $386.67 \mathrm{ab}$ & $6,49 a b$ \\
\hline C+ H.Das-3383 & $3.49 \mathrm{bc}$ & $15.01 \mathrm{~cd}$ & 275.00 ef & 4,61 ef \\
\hline $\mathrm{C}+2 \mathrm{~B}-604$ & $3.03 \mathrm{c}$ & $13.02 \mathrm{~d}$ & $261.67 \mathrm{f}$ & $4,39 \mathrm{f}$ \\
\hline $\mathrm{C}+$ Insignia-105 & $3.50 \mathrm{bc}$ & $15.98 \mathrm{bc}$ & 275.00 ef & 4,63 ef \\
\hline $\mathrm{C}+$ Dekalb-7500 & $3.76 \mathrm{bc}$ & $16.72 \mathrm{abc}$ & 288.33 def & 4,84 def \\
\hline CV(\%) & 11.86 & 7.17 & 5.68 & 5.60 \\
\hline
\end{tabular}

Los valores con letras similares no representan diferencias estadísticas significativas al nivel de confianza del $95 \%$, por el procedimiento de comparación múltiple de Tukey. Edáfica (E); Foliar (F); Control (C); Días (d).

Indudablemente el rendimiento (R) de los híbridos incrementó por la aplicación del fertilizante edáfica+foliar, es evidente en los híbridos Insignia-105 y 2B-604 con 7,18 y 7,19 t/ha (Tabla 4). La productividad se incrementó bajo las aplicaciones de abonos foliares al maíz, realizada antes de los días de floración, esto mejoró sustancialmente en sus aspectos fisiológicos con el incremento de sus caracteres: AIM, LM y PMS, como resultado mayor productividad. Por la incorporación de reguladores de crecimiento vegetal como, giberelinas y auxinas en los fertilizantes foliares que benefician a la planta. Los híbridos bajo fertilización foliar desarrollaron abundante follaje, obteniendo una mejor capacidad fotosintética que incrementa la producción de almidones y azucares. Beyranvand et al., (2013), demuestran que el peso de semilla se incrementa por la capacidad fotosintética del maíz. Los fertilizantes foliares BIOTEK y EVERGREEN, contiene reguladores de crecimiento vegetal que beneficiaron directamente al desarrollo de la mazorca en DM y LM. La aplicación de ácido giberélico (GA) y la auxina ácido indolbutírico (IBA) a 100 $\mathrm{mg} / \mathrm{L}$ durante el desarrollo fisiológico antes de su floración, incrementa en tamaño de mazorca y productividad de semillas con 17.2 t/ha (Ghodrat et al., 2012).

La aplicación de reguladores de crecimiento de plantas (RCPs), incrementa los parámetros a productividad por: índice de área foliar, porcentaje de asimilación neta y duración de su área foliar es mejorada (Thavaprakaash et al., 2007).
En trigo se obtiene alta productiva por la aplicación de altos niveles de auxinas (Eradatmand et al., 2011). También se ha observado el empleo de ácido giberélico y auxina tienen efecto en rendimiento en maíz (Ahmad et al., 2008). Ratificado por Bautista (2002), la fertilización foliar es una alternativa para incrementar el rendimiento mediante la aportación y rápida asimilación de los nutrimentos durante el desarrollo del maíz que sirve de complemento a la fertilización edáfica.

En la actualidad el uso indebido de pesticidas y agroquímicos inciden en la pérdida de la biodiversidad microbiana. Las rizobacterias aumentan la disponibilidad de $\mathrm{P}$, estas liberan compuestos de crecimiento como: (Giberelina, Citoquinina y Auxinas), que son eficaces en el crecimiento de la planta (Ortas et al., 1996). La disminución de la biodiversidad microbiana, se requiere de aplicaciones de abonos foliares y bio-fertilizantes que contribuyan a mejorar el aspecto fisiológico del maíz, el empleo de BIOTEK y ECO-HUM DX contiene reguladores de crecimiento (Citoquininas, Auxinas y Giberelinas), que favorecieron al desarrollo del maíz.

\section{Conclusiones}

T as aportaciones de los macronutriente, micronutrientes Ly reguladores de crecimiento por la fertilización edáfica+foliar, incrementan los caracteres fisiológicos de los híbridos de maíz. Reflejado por una mayor productividad del 
híbrido Insignia-105 con (7.19 t/ha). La adición de estos abonos foliares resaltarón su potencial genético en todos los híbridos, reflejando un efecto sinérgico en la planta por asimilación de nutrientes a través de sus hojas. La implementación de la fertilización edáfica+foliar en el cultivo de maíz reduce el riesgo de producción, pero aumenta los costos de operación.

\section{Referencias}

Ahmad, R., Arshad, Z., Zahire, Z., Naveed, M., Khalid, M. \& Asghar, H. (2008). Integrating- enriched compost with biologically active substances for improving growth and yield of cereals. Pakistan Journal Botany. 40 (1), 283293. Recuperado de http://pakbs.org/pjbot/PDFs/40(1)/ PJB40(1)283.pdf

Banks, L.W. (2004). Effect of timing of foliar zinc fertilizer on yieldcomponent of soybeans. Australian. Journal Experimental Agriculture Animal Husbandry. 22 (116), 226-231. Recuperado de Doi: http://dx.doi.org/10.1071/ EA9820226

Bautista, R. (2002). Efecto de la fertilización foliar en el rendimiento y calidad efecto de la fertilización foliar en el rendimiento y calidad. Revistate Fitotecnica Mexicana. 25 (4), 419 - 426. Recuperado de http://www. revistafitotecniamexicana.org/documentos/25-4/12a.pdf

Beyranvand, B., Farnia, A., Nakhjavan, S. \& Shaban, M. (2013). Response of yield and yield components of maize (Zea mayz L.) to different bio fertilizers. International journal of Advanced Biological and Biomedical Research. 1(9),1068-1077. Recuperado de http:/www.ijabbr.com/ article_7870_1266.html

Bukvić, G., Antunović1, M., Popović, S. \& Rastija, M. (2003). Effect of $\mathrm{P}$ and $\mathrm{Zn}$ fertilisation on biomass yield and its uptake by maize lines (Zea mays L.). Plant soil environ. 49 (11), 505-510. Recuperado de http://citeseerx.ist.psu. edu/viewdoc/download?doi=10.1.1.497.1479\&rep=rep1 \&type $=\mathrm{pdf}$

Dunja, M. (2000). Fertilización del cultivo maíz. Revista de difusión de tecnología agrícola y pesquera del FONAIAPDIVULGA. 65.

Eradatmand, D. \& Houshmandfar, A. (2011). Dry matter accumulation and auxin levels within developing grains of different durum wheat genotypes. Advances in Environmental Biology. 5 (4), 678682. Recuperado de http://scinet.dost.gov.ph/union/ Downloads/678-682_225063.pdf

Farmagro (2015). Formato para agricultores Maiceros. Quevedo.

Fecenko, J. \& Ložek, O. (1998). Maize grain yield formation in dependence on applied zinc doses and its content in soil. Rostlinná Výroba. 44 (5), 15-18. Recuperado de http://agris.fao.org/agris-search/search. do? recordID $=$ CZ1998000253

Figliolia, A., Benedetti, A., Izza, C., Indiati, R., Rea, E.,
Alianiello, F., Canali. S., Biondi, F. A., Pierandrei, F. \& Moretti, R. (1994) Effects of fertilization with humic acid on soils and plant metabolism: A multidisciplinary approach. Note I: Crop production, in: Humic Substances in the Global Environment and Implications on Human Health Proceedings of the 6th International Meeting of the International Humic Substances Society, Elsevier Science Publishers, Amsterdam, The Netherlands. pp. 579-584.

Fu Jiu, C., Dao Qi, Y., \& Quing Sheng, W. (1995) Physiological effects of

humic acid on drought resistance of wheat (in Chinese), Yingyong Shengtai Xuebao. 6 (1), 363-367. Recuperado de http://agris.fao.org/agris-search/search. do? recordID $=$ CN9611622

Ghodrat, V., Mohammad. J. R., Mohammad. S. T., \& Karampour, A. (2012). Yield and yield components of corn (Zea mays L.) in response to foliar application with indole butyric acid and gibberellic acid. AmericanEurasian Journal of Agricultural \& Environmental. 12 (9), 1246-1251. Recuperado de http://www.idosi.org/ aejaes/jaes12(9)12/19.pdf

INIAP. 2010. Hibridos de maiz para la zona Central del Litoral. Programa de maiz, ETT-Pichilingue.

Di Rienzo, J.A., Casanoves, F., Balzarini M.G., Gonzalez, L., Tablada, M. \& Robledo, C.W. (2008). InfoStat, versión 2008. Grupo InfoStat, FCA, Universidad Nacional de Cordoba, Argentina.

Kariali. E. \& Mohapatra. P. K. (2007). Hormonal regulation of tiller dynamics in differntially-tillering rice cultivares. Plant Growth Regulation. 53 (3), 215-223. Recuperado de http://link.springer.com/article/10.1007/s10725-0079221-z

Kefyalew, G., Martin, K., Freeman, K., Mosali, J., Teal. R., Raun.W., Moges, S. \& Arnall, D. (2007). Determination of Optimum Rate and Growth Stage for Foliar $\square$ Applied Phosphorus in Corn. Communications in Soil Science and Plant Analysis. 38 (9), 1137-1154. Recuperado de http://www.tandfonline.com/doi/ abs/10.1080/00103620701328016

Khaliq, A. \& Sanders, F. (2000). Effects of vesicular arbuscular mycorrhizal inoculation on the yield and phosphorus uptake of field grown barley. Soil Biology and Biochemistry. 32 (11), 1691 -1696. Recuperado de http:/www.sciencedirect.com/science/article/pii/ S0038071700000869

Law, K. \& Law, J. (2009). The Performance of Zea mays as Influenced by NPK Fertilizer Application. Notulae Scientia Biologicae. 1 (4), 59-62. Recuperado de http:// www.notulaebiologicae.ro/

Ling, L. \& Silberbush, M. (2002). Response of maize to foliar vs. soil application of nitrogen phosphorus-potassium fertilizers. Journal of plant nutrition. 25 (11), 23332342. Recuperado de http://www.tandfonline.com/doi/ 
abs/10.1081/PLN-120014698

Ministerio de Agricultura, Ganadería, Acuacultura y Pesca. (MAGAP). (2012). Consultado en agosto del 2015. En sinagap.agricultura.gob.ec. Recuperado de http://sinagap. agricultura.gob.ec/maiz-suave-seco-2.

Monteros Guerrero, A. (2015). Rendimiento de maíz duro seco verano. SINAGAP. Recuperado de http://sinagap. agricultura.gob.ec/pdf/estudios_agroeconomicos/ rendimiento_maiz_duro_seco_verano2015.pdf.

Robledo, R., Zamudio, B., Espinosa, A., Turrent, A., Cárdenas, A., López, C., Arteaga, I. \& Valdivia, R. (2015). Rendimiento de maíces nativos e híbridos en diferente fecha de siembra y sus unidades calor. Revista Mexicana de Ciencias Agrícolas. 6 (1), 33-43. Recuperado de http:// www.scielo.org.mx/pdf/remexca/v6n1/v6n1a4.pdf

Shahid, I., Haroon, K., Muhammad, Z., Muhammad W. M., \& Hafi M. J. (2014). The effects of nitrogen fertilization strategies on the productivity of maize (Zea mays L.) hybrids. Zemdirbyste-Agriculture.101 (3), 249-256. Recuperado de searchgate.net/profile/ Shahid_Iqbal19/publication/260943917_The effects_of_nitrogen_fertilization_strategies_on_ the productivity of maize (Zea maysL.) hybrids/ links/543578140cf2dc341db2a6b8.pdf

Suwanarit, A. \& Sestapukdee, M. (1989). Stimulating effects of foliar k-fertilizer applied at the appropriate stage of development of maize: a new way to increase yield and improve quality. Plant soil. 120 (1), 111-124. Recuperado de http://ink.springer.com/article/10.1007/BF02370297

Thavaprakaash, N., Velayudham, K. \& Gurusamy, L. (2007).
Influence of herbicides growth regulators on growth and yield of Baby Corn (Zea mays L.). International Journal Agricultural Research. 2 (8), 731-735. Recuperado de http://docsdrive.com/pdfs/academicjournals/ ijar/2007/731-735.pdf

Ortas, I. (1996). The influence of use of different rates of mycorrhizal inoculum on root infection, plant growth, and phosphorus uptake. Soil Science and Plant Annual. 27 (18), 2935-2946. Recuperado de http://www.tandfonline. com/doi/abs/10.1080/00103629609369753

Potarzycki, J. \& Grzebisz, W. (2009). Effect of zinc foliar application on grain yield of maize

and its yielding components. Plant soil environment. 55 (12), 519-527. Recuperado de http://www.inea_00fcr.user. icpnet.pl/pdf/Plant_Soil_Environ_2009.pdf

Prochazka, S. (1978). Effect of indol-3-acetic acid on the translocation of assimilates in winter wheat (Triticum aestivum L.) in the period of kernel formation. Acta University of Agriculture Brno. 26 (3), 99-104. Recuperado de http://agris.fao.org/agris-search/search. do? recordID $=\mathrm{CZ} 19800469463$

Zeidan, M. S., Mohamed, M. F y Hamouda, H. A. (2010). Effect of foliar fertilization of $\mathrm{Fe}, \mathrm{Mn}$ and $\mathrm{Zn}$ on wheat yield and quality in low Sandy soils fertility. World Journal Agricultural Sciences. 6 (6), 696-699. Recuperado de http://idosi.org/wjas/wjas6(6)/11.pdf 